\title{
A Synthesis of (+)-Saxitoxin
}

\section{Supplementary Material}

(7 pages)

James J. Fleming and J. Du Bois*

Department of Chemistry

Stanford University

Stanford, CA 94305-5080 
General. Nuclear magnetic resonance (NMR) spectra were acquired on a Varian Inova spectrometer operating at 400 or 500 and 100 or $125 \mathrm{MHz}$ for ${ }^{1} \mathrm{H}$ and ${ }^{13} \mathrm{C}$, respectively, and are referenced internally according to residual solvent signals. Data for ${ }^{1} \mathrm{H}$ NMR are recorded as follows: chemical shift $(\delta, \mathrm{ppm})$, multiplicity (s, singlet; $\mathrm{d}$, doublet; t, triplet; q, quartet; quint, quintet; m, multiplet; br, broad), integration, coupling constant (Hz). Data for ${ }^{13} \mathrm{C}$ NMR are reported in terms of chemical shift ( $\delta$, ppm). Infrared (IR) spectra were recorded as either thin films using $\mathrm{NaCl}$ plates or as $\mathrm{KBr}$ pellets on a Thermo-Nicolet $300 \mathrm{FT}$-IR spectrometer and are reported in frequency of absorption. Optical rotation data were obtained from samples loaded into a $50 \mathrm{~mm}$ cell on a Jasco DIP-1000 digital polarimeter operating at the Na D-line. High resolution mass spectra were obtained from the Vincent Coates Foundation Mass Spectrometry Laboratory at Stanford University.

\section{Characterization Data for Selected Compounds}

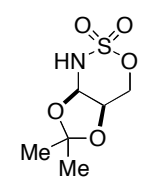

TLC $\mathrm{R}_{f}=0.48\left(1: 1\right.$ hexanes/EtOAc); mp $96-97{ }^{\circ} \mathrm{C} ;[\alpha]_{\mathrm{Na}}-44.1^{\circ}(\mathrm{c}=1.50, \mathrm{MeOH}) ;{ }^{1} \mathrm{H}$ NMR $\left(\mathrm{CD}_{3} \mathrm{OD}, 500 \mathrm{MHz}\right) \delta$ $5.42(\mathrm{~d}, 1 \mathrm{H}, J=5.3 \mathrm{~Hz}), 4.59-4.58(\mathrm{~m}, 2 \mathrm{H}), 4.31(\mathrm{ddd}, 1 \mathrm{H}, J=5.3,1.6,1.6 \mathrm{~Hz}), 1.54(\mathrm{~s}, 3 \mathrm{H}), 1.35(\mathrm{~s}, 3 \mathrm{H}) \mathrm{ppm} ;{ }^{13} \mathrm{C}$ NMR $\left(\mathrm{CD}_{3} \mathrm{OD}, 125 \mathrm{MHz}\right) \delta 111.5,85.6,71.7,71.5,26.9,25.6 \mathrm{ppm}$; IR (KBr pellet) v 3260, 2997, 2979, 1456, $1390,1379,1354,1245,1180,1071 \mathrm{~cm}^{-1}$; HRMS $\left(\mathrm{ES}^{+}\right)$calcd for $\mathrm{C}_{6} \mathrm{H}_{11} \mathrm{NO}_{5} \mathrm{~S} 209.0358$ found $232.0246\left(\mathrm{MNa}^{+}\right)$.

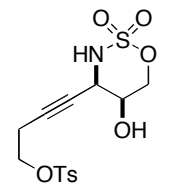

TLC $\mathrm{R}_{f}=0.34\left(2: 3\right.$ hexanes/EtOAc); mp $112-114^{\circ} \mathrm{C} ;[\alpha]_{\mathrm{Na}}-10.4^{\circ}(\mathrm{c}=2.40, \mathrm{MeOH}) ;{ }^{1} \mathrm{H}$ NMR $\left(\mathrm{CD}_{3} \mathrm{OD}, 500 \mathrm{MHz}\right)$ $\delta 7.85(\mathrm{~d}, 2 \mathrm{H}, J=8.3 \mathrm{~Hz}), 7.50$ (d, $2 \mathrm{H}, J=8.5 \mathrm{~Hz}), 4.68(\mathrm{dd}, 1 \mathrm{H}, J=12.3,1.4 \mathrm{~Hz}), 4.56$ (br d, $1 \mathrm{H}, J=1.8 \mathrm{~Hz}), 4.42$ $(\mathrm{dd}, 1 \mathrm{H}, J=12.3,2.2 \mathrm{~Hz}), 4.12(\mathrm{dd}, 2 \mathrm{H}, J=6.5,6.5 \mathrm{~Hz}), 3.61(\mathrm{dd}, 1 \mathrm{H}, J=4.3,1.8 \mathrm{~Hz}), 2.62(\mathrm{ddd}, 2 \mathrm{H}, J=6.5,6.5$, $2.1 \mathrm{~Hz}), 2.50(\mathrm{~s}, 3 \mathrm{H}) \mathrm{ppm} ;{ }^{13} \mathrm{C}$ NMR $\left(\mathrm{d}_{6}\right.$-acetone, $\left.125 \mathrm{MHz}\right) \delta 146.0,133.9,130.9,128.7,82.2,77.2,76.6,68.7$, 63.2, 53.3, 21.5, 20.0 ppm; IR (KBr pellet) v 3477, 3220, 2260, 1380, 1353, 1175, 1012, $963 \mathrm{~cm}^{-1}$; HRMS (ES ${ }^{+}$) calcd for $\mathrm{C}_{14} \mathrm{H}_{17} \mathrm{NO}_{7} \mathrm{~S}_{2} 375.0446$ found $398.0334\left(\mathrm{MNa}^{+}\right)$.

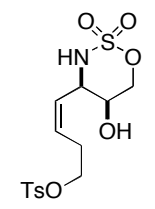

TLC $\mathrm{R}_{f}=0.34$ (2:3 hexanes/EtOAc); ${ }^{1} \mathrm{H}$ NMR $\left(\mathrm{CDCl}_{3}, 400 \mathrm{MHz}\right) \delta$ 7.78-7.76 (m, 2H), 7.38-7.38 (m, 2H), 5.72$5.62(\mathrm{~m}, 2 \mathrm{H}), 4.90(\mathrm{br} \mathrm{s}, 1 \mathrm{H}), 4.80(\mathrm{dd}, 1 \mathrm{H}, J=12.4,1.4 \mathrm{~Hz}), 4.56(\mathrm{br} \mathrm{d}, 1 \mathrm{H}, J=5.3 \mathrm{~Hz}), 4.51(\mathrm{dd}, 1 \mathrm{H}, J=12.4,2.0$ $\mathrm{Hz}), 4.14-4.02(\mathrm{~m}, 2 \mathrm{H}), 3.69(\mathrm{dd}, 1 \mathrm{H}, J=3.2,1.8 \mathrm{~Hz}), 2.62-2.50(\mathrm{~m}, 1 \mathrm{H}), 2.49-2.42(\mathrm{~m}, 1 \mathrm{H}), 2.46(\mathrm{~s}, 3 \mathrm{H}) \mathrm{ppm} ;{ }^{13} \mathrm{C}$ NMR $\left(\mathrm{CDCl}_{3}, 100 \mathrm{MHz}\right) \delta 145.3,132.6,130.6,130.1,127.9,126.9,76.7,69.1,63.1,56.6,28.0,21.8 \mathrm{ppm}$; IR (thin film) $\vee 3523,3261,2960,1598,1427,1356,1188,1175,1020,917 \mathrm{~cm}^{-1}$; HRMS $\left(\mathrm{ES}^{\dagger}\right)$ calcd for $\mathrm{C}_{14} \mathrm{H}_{19} \mathrm{NO}_{7} \mathrm{~S}_{2}$ 377.0603 found $400.0500\left(\mathrm{MNa}^{+}\right)$.<smiles>NCC/C=C\C(O)C(O)COC(=O)O</smiles>

TLC $\mathrm{R}_{f}=0.62\left(2: 3\right.$ hexanes/EtOAc); $[\alpha]_{\mathrm{Na}}+54.1^{\circ}\left(\mathrm{c}=2.60, \mathrm{CHCl}_{3}\right) ;{ }^{1} \mathrm{H} \mathrm{NMR}\left(\mathrm{CDCl}_{3}, 500 \mathrm{MHz}\right) \delta$ 7.24-7.22 (m, $2 \mathrm{H}), 6.86-6.84(\mathrm{~m}, 2 \mathrm{H}), 5.90(\mathrm{dddd}, 1 \mathrm{H}, J=11.1,9.6,1.5,1.5 \mathrm{~Hz}), 5.80-5.75(\mathrm{~m}, 1 \mathrm{H}), 4.71(\mathrm{dd}, 1 \mathrm{H}, J=11.8,2.4$ 
$\mathrm{Hz}), 4.56(\mathrm{dd}, 1 \mathrm{H}, J=9.5,2.8 \mathrm{~Hz}), 4.48(\mathrm{dd}, 1 \mathrm{H}, J=11.8,4.4 \mathrm{~Hz}), 4.40(\mathrm{~d}, 1 \mathrm{H}, J=15.4 \mathrm{~Hz}), 4.31(\mathrm{~d}, 1 \mathrm{H}, J=15.4$ $\mathrm{Hz}), 3.82-8.79(\mathrm{~m}, 1 \mathrm{H}), 3.80(\mathrm{~s}, 3 \mathrm{H}), 3.34-3.30(\mathrm{~m}, 1 \mathrm{H}), 3.24(\mathrm{ddd}, 1 \mathrm{H}, J=12.2,8.2,5.6 \mathrm{~Hz}), 2.45(\mathrm{~d}, 1 \mathrm{H}, J=8.7$ $\mathrm{Hz}), 2.29-2.22(\mathrm{~m}, 1 \mathrm{H}), 2.22-2.14(\mathrm{~m}, 1 \mathrm{H}) \mathrm{ppm} ;{ }^{13} \mathrm{C} \mathrm{NMR}\left(\mathrm{CDCl}_{3}, 125 \mathrm{MHz}\right) \delta$ 159.1, 134.2, 129.6, 128.6, 124.6, 113.9, 75.2, 65.0, 59.0, 55.2, 50.3, 50.1, 27.6 ppm; IR (thin film) v 3528, 2937, 2100, 1613, 1514, 1377, 1247, 1178, $1035,969 \mathrm{~cm}^{-1}$; HRMS (ES ${ }^{+}$) calcd for $\mathrm{C}_{15} \mathrm{H}_{20} \mathrm{~N}_{4} \mathrm{O}_{5} \mathrm{~S} 368.1154$ found $391.1052\left(\mathrm{MNa}^{+}\right)$.

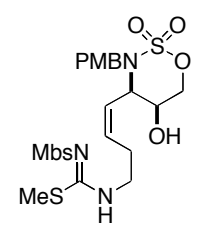

TLC $\mathrm{R}_{f}=0.43\left(1: 2\right.$ hexanes/EtOAc); ${ }^{1} \mathrm{H}$ NMR $\left(\mathrm{CDCl}_{3}, 500 \mathrm{MHz}\right) \delta 7.95$ (br s, 1H), 7.81-7.78 (m, 2H), 7.25-7.22 $(\mathrm{m}, 2 \mathrm{H}), 6.96-6.93(\mathrm{~m}, 2 \mathrm{H}), 6.85-6.82(\mathrm{~m}, 2 \mathrm{H}), 6.01(\mathrm{dd}, 1 \mathrm{H}, J=10.7,9.5 \mathrm{~Hz}), 5.70-5.65(\mathrm{~m}, 1 \mathrm{H}), 4.70(\mathrm{dd}, 1 \mathrm{H}, J=$ $11.8,2.4 \mathrm{~Hz}), 4.61(\mathrm{dd}, 1 \mathrm{H}, J=9.0,2.3 \mathrm{~Hz}), 4.50(\mathrm{dd}, 1 \mathrm{H}, J=12.0,4.6 \mathrm{~Hz}), 4.42(\mathrm{~d}, 1 \mathrm{H}, J=15.5 \mathrm{~Hz}), 4.36(\mathrm{~d}, 1 \mathrm{H}$, $J=15.6 \mathrm{~Hz}), 3.97-3.94(\mathrm{br} \mathrm{m}, 1 \mathrm{H}), 3.85(\mathrm{~s}, 3 \mathrm{H}), 3.78(\mathrm{~s}, 3 \mathrm{H}), 3.33(\mathrm{ddd}, 1 \mathrm{H}, J=13.2,10.8,5.6 \mathrm{~Hz}), 3.20-3.14(\mathrm{~m}$, $1 \mathrm{H}), 2.95(\mathrm{br} \mathrm{d}, 1 \mathrm{H}, J=6.0 \mathrm{~Hz}), 2.39-2.28(\mathrm{~m}, 1 \mathrm{H}), 2.35(\mathrm{~s}, 3 \mathrm{H}), 2.13-2.09(\mathrm{~m}, 1 \mathrm{H}) \mathrm{ppm} ;{ }^{13} \mathrm{C} \mathrm{NMR}\left(\mathrm{CDCl}_{3}, 125\right.$ MHz) $\delta 169.1,162.6,159.1,134.0,132.8,129.5,129.0,128.1,126.4,113.9,113.8,75.2,64.0,58.5,55.6,55.3$, 50.6, 42.9, 27.6, $14.2 \mathrm{ppm}$; IR (thin film) v 3453, 3301, 2936, 1571, 1513, 1498, 1376, 1258, 1180, 1137, 1079, $1031,970 \mathrm{~cm}^{-1}$; HRMS (ES $\left.{ }^{+}\right)$calcd for $\mathrm{C}_{24} \mathrm{H}_{31} \mathrm{~N}_{3} \mathrm{O}_{8} \mathrm{~S}_{3} 585.1237$ found $608.1185\left(\mathrm{MNa}^{+}\right)$.

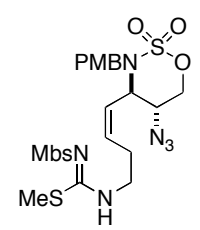

TLC $\mathrm{R}_{f}=0.33\left(1: 1\right.$ hexanes/EtOAc); $[\alpha]_{\mathrm{Na}}+93.5^{\circ}\left(\mathrm{c}=2.00, \mathrm{CHCl}_{3}\right) ;{ }^{1} \mathrm{H}$ NMR $\left(\mathrm{CDCl}_{3}, 500 \mathrm{MHz}\right) \delta 8.10-8.05(\mathrm{br}$ $\mathrm{m}, 1 \mathrm{H}), 7.85-7.82(\mathrm{~m}, 2 \mathrm{H}), 7.31-7.28(\mathrm{~m}, 2 \mathrm{H}), 6.98-6.95(\mathrm{~m}, 2 \mathrm{H}), 6.92-6.89(\mathrm{~m}, 2 \mathrm{H}), 6.05(\mathrm{dd}, 1 \mathrm{H}, J=10.9,10.0$ $\mathrm{Hz}), 5.71(\mathrm{dddd}, 1 \mathrm{H}, J=10.9,8.7,6.6,1.0 \mathrm{~Hz}), 4.86(\mathrm{dd}, 1 \mathrm{H}, J=12.3,2.9 \mathrm{~Hz}), 6.88(\mathrm{~d}, 1 \mathrm{H}, J=14.4 \mathrm{~Hz}), 4.53$ (ddd, $1 \mathrm{H}, J=12.4,4.8,1.3 \mathrm{~Hz}), 4.30(\mathrm{dd}, 1 \mathrm{H}, J=9.6,4.6 \mathrm{~Hz}), 4.21(\mathrm{~d}, 1 \mathrm{H}, J=14.4 \mathrm{~Hz}), 3.88(\mathrm{~s}, 3 \mathrm{H}), 3.83(\mathrm{~s}, 3 \mathrm{H}), 3.54$ (ddd, $1 \mathrm{H}, J=4.6,4.6,2.9 \mathrm{~Hz}), 3.33-3.27(\mathrm{~m}, 1 \mathrm{H}), 3.25-3.20(\mathrm{~m}, 1 \mathrm{H}), 2.36(\mathrm{~s}, 3 \mathrm{H}), 2.21-2.07(\mathrm{~m}, 2 \mathrm{H}) \mathrm{ppm} ;{ }^{13} \mathrm{C}$ NMR $\left(\mathrm{CDCl}_{3}, 125 \mathrm{MHz}\right) \delta 168.8,162.5,159.6,134.2,132.7,130.3,130.2,128.2,126.6,114.1,113.9,71.2,58.3$, 56.1, 55.6, 55.3, 50.8, 42.8, 27.3, $14.1 \mathrm{ppm}$; IR (thin film) v 3299, 2933, 2112, 1573, 1513, 1367, 1307, 1258, 1175, $1139,1079,1028,834 \mathrm{~cm}^{-1}$; HRMS $\left(\mathrm{ES}^{+}\right)$calcd for $\mathrm{C}_{24} \mathrm{H}_{30} \mathrm{~N}_{6} \mathrm{O}_{7} \mathrm{~S}_{3} 610.1338$ found $633.1226\left(\mathrm{MNa}^{+}\right)$.

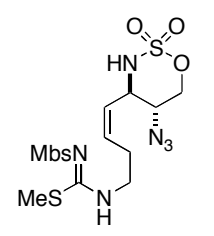

TLC $\mathrm{R}_{f}=0.42\left(9: 1 \mathrm{CH}_{2} \mathrm{Cl}_{2} / \mathrm{EtOAc}\right) ;{ }^{1} \mathrm{H}$ NMR $\left(\mathrm{CDCl}_{3}, 500 \mathrm{MHz}\right) \delta$ 7.91-7.88 (m, 2H), 7.87 (br s, 1H), 6.99-6.96 (m, 2H), 5.87-5.82 (m, 1H), 5.69 (br t, $1 \mathrm{H}, J=9.5 \mathrm{~Hz}), 5.52(\mathrm{~d}, 1 \mathrm{H}, J=7.8 \mathrm{~Hz}), 4.57(\mathrm{dd}, 1 \mathrm{H}, J=11.6,5.1 \mathrm{~Hz})$, 4.49 (dd, $1 \mathrm{H}, J=11.6,10.4 \mathrm{~Hz}), 4.41-4.36(\mathrm{~m}, 1 \mathrm{H}), 3.90$ (s, 3H), 3.68 (ddd, $1 \mathrm{H}, J=10.2,10.2,5.3 \mathrm{~Hz}), 3.60-3.54$ $(\mathrm{m}, 1 \mathrm{H}), 3.35-3.31(\mathrm{~m}, 1 \mathrm{H}), 2.86-2.78(\mathrm{~m}, 1 \mathrm{H}), 2.42-2.38(\mathrm{~m}, 1 \mathrm{H}), 2.40(\mathrm{~s}, 3 \mathrm{H}) \mathrm{ppm} ;{ }^{13} \mathrm{C} \mathrm{NMR}\left(\mathrm{CDCl}_{3}, 125 \mathrm{MHz}\right) \delta$ $169.3,162.6,133.9,128.3,128.2,127.4,113.9,71.0,56.3,55.6,55.5,42.9,27.7,14.1 \mathrm{ppm}$; IR (thin film) v 3299 , 2932, 2114, 1572, 1498, 1433, 1370, 1258, 1189, 1139, $1080 \mathrm{~cm}^{-1}$; HRMS (ES ${ }^{+}$) calcd for $\mathrm{C}_{16} \mathrm{H}_{22} \mathrm{~N}_{6} \mathrm{O}_{6} \mathrm{~S}_{3} 490.0763$ found $513.0661\left(\mathrm{MNa}^{+}\right)$. 


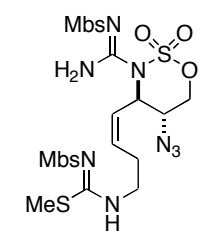

TLC $\mathrm{R}_{f}=0.37\left(2: 3\right.$ hexanes/EtOAc); $[\alpha]_{\mathrm{Na}}+71.0^{\circ}\left(\mathrm{c}=3.40, \mathrm{CHCl}_{3}\right) ;{ }^{1} \mathrm{H} \mathrm{NMR}\left(\mathrm{CDCl}_{3}, 500 \mathrm{MHz}\right) \delta 8.04(\mathrm{br} \mathrm{s}, 1 \mathrm{H})$, 7.88-7.82 (m, 4H), 7.00-6.95 (m, 4H), $6.04(\mathrm{t}, 1 \mathrm{H}, J=10.4 \mathrm{~Hz}), 5.99-5.93(\mathrm{~m}, 1 \mathrm{H}), 5.70-5.64(\mathrm{~m}, 1 \mathrm{H}), 5.04(\mathrm{dd}, 1 \mathrm{H}$, $J=12.2,1.6 \mathrm{~Hz}$ ), 4.60 (ddd, $1 \mathrm{H}, J=12.5,2.7,1.7 \mathrm{~Hz}), 3.99$ (br d, $1 \mathrm{H}, J=2.3 \mathrm{~Hz}$ ), $3.88(\mathrm{~s}, 3 \mathrm{H}), 3.87$ (s, $3 \mathrm{H}), 3.50-$ $3.45(\mathrm{~m}, 1 \mathrm{H}), 3.31-3.25(\mathrm{~m}, 1 \mathrm{H}), 2.72-2.64(\mathrm{~m}, 1 \mathrm{H}), 2.39-2.33(\mathrm{~m}, 1 \mathrm{H}), 2.38(\mathrm{~s}, 3 \mathrm{H}) \mathrm{ppm} ;{ }^{13} \mathrm{C} \mathrm{NMR}\left(\mathrm{CDCl}_{3}, 125\right.$ MHz) $\delta 168.9,162.8,162.5,151.9,134.1,133.7,130.3,128.3,128.2,125.3,114.0,113.9,73.2,57.5,56.2,55.6$, $55.5,42.8,27.8,14.1 \mathrm{ppm}$; IR (thin film) v 3447, 3317, 2945, 2114, 1626, 1596, 1577, 1499, 1419, 1310, 1260, $1192,1141,1078,1025 \mathrm{~cm}^{-1}$.

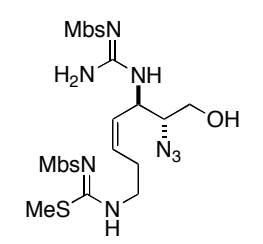

TLC $\mathrm{R}_{f}=0.36\left(2: 7\right.$ hexanes/EtOAc); $[\alpha]_{\mathrm{Na}}-103^{\circ}\left(\mathrm{c}=2.20, \mathrm{CHCl}_{3}\right) ;{ }^{1} \mathrm{H} \mathrm{NMR}\left(\mathrm{CDCl}_{3}, 500 \mathrm{MHz}\right) \delta 7.87-7.84(\mathrm{~d}$, 2H), $7.81(\mathrm{~d}, 2 \mathrm{H}, J=8.9 \mathrm{~Hz}), 7.78(\mathrm{br} \mathrm{s}, 1 \mathrm{H}), 6.97(\mathrm{~d}, 2 \mathrm{H}, J=8.9 \mathrm{~Hz}), 6.93(\mathrm{~d}, 2 \mathrm{H}, J=9.0 \mathrm{~Hz}), 6.70$ (br s, 2H), 5.97 (br s, 1H), $5.71(\mathrm{t}, 1 \mathrm{H}, J=9.3 \mathrm{~Hz}), 5.61-5.56(\mathrm{~m}, 1 \mathrm{H}), 4.79-4.76(\mathrm{br} \mathrm{m}, 1 \mathrm{H}), 3.87(\mathrm{~s}, 3 \mathrm{H}), 3.85(\mathrm{~s}, 3 \mathrm{H}), 3.73(\mathrm{br} \mathrm{s}$, $2 \mathrm{H}), 3.40(\mathrm{br} \mathrm{s}, 1 \mathrm{H}), 3.27-3.22(\mathrm{~m}, 2 \mathrm{H}), 2.36(\mathrm{~s}, 3 \mathrm{H}), 2.26-2.22(\mathrm{~m}, 2 \mathrm{H}) \mathrm{ppm} ;{ }^{13} \mathrm{C} \mathrm{NMR}\left(\mathrm{CDCl}_{3}, 125 \mathrm{MHz}\right) \delta 169.5$, $162.7,162.3,155.8,134.9,133.5,132.0,129.8,128.1,128.0,114.0,113.8,65.4,61.3,55.6,55.5,47.3,43.0,27.5$, $14.2 \mathrm{ppm}$; IR (thin film) v 3444, 3338, 2944, 2103, 1596, 1577, 1533, 1499, 1258, 1133, 1083, $833 \mathrm{~cm}^{-1}$; HRMS $\left(\mathrm{ES}^{+}\right)$calcd for $\mathrm{C}_{24} \mathrm{H}_{32} \mathrm{~N}_{8} \mathrm{O}_{7} \mathrm{~S}_{3} 640.1556$ found $663.1437\left(\mathrm{MNa}^{+}\right)$.

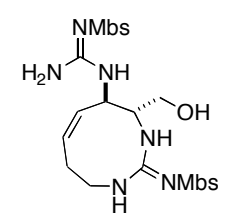

TLC $\mathrm{R}_{f}=0.28\left(20: 1 \mathrm{CH}_{2} \mathrm{Cl}_{2} / \mathrm{MeOH}\right) ; \mathrm{mp} 147-150{ }^{\circ} \mathrm{C} ;[\alpha]_{\mathrm{Na}}+91.0^{\circ}(\mathrm{c}=2.60, \mathrm{MeOH}) ;{ }^{1} \mathrm{H} \mathrm{NMR}\left(\mathrm{CD}_{3} \mathrm{CN}, 500\right.$ $\left.\mathrm{MHz}, 70{ }^{\circ} \mathrm{C}\right) \delta$ 7.81-7.75 (m, 4H), 7.07-7.01 (m, 4H), $6.73(\mathrm{br} \mathrm{s}, 1 \mathrm{H}), 6.25$ (br s, 2H), 5.76 (br s, $\left.1 \mathrm{H}\right), 5.00$ (br s, $1 \mathrm{H}), 4.87-4.83(\mathrm{~m}, 1 \mathrm{H}), 4.69(\mathrm{t}, 1 \mathrm{H}, J=10.0 \mathrm{~Hz}), 3.97-3.87(\mathrm{br} \mathrm{m}, 1 \mathrm{H}), 3.90(\mathrm{~s}, 3 \mathrm{H}), 3.89(\mathrm{~s}, 3 \mathrm{H}), 3.78(\mathrm{br} \mathrm{d}, 1 \mathrm{H}, J$ $=11.7 \mathrm{~Hz}), 3.68(\mathrm{br} \mathrm{d}, 1 \mathrm{H}, J=11.9 \mathrm{~Hz}), 3.51-3.43(\mathrm{~m}, 2 \mathrm{H}), 3.27($ br s, $1 \mathrm{H}), 2.49(\mathrm{br} \mathrm{s}, 1 \mathrm{H}), 2.04-2.00($ br m, $1 \mathrm{H})$ ppm; IR (KBr pellet) v 3335, 2943, 1597, 1531, 1499, 1257, 1131, 1080, $834 \mathrm{~cm}^{-1}$; HRMS (ES ${ }^{+}$) calcd for $\mathrm{C}_{23} \mathrm{H}_{30} \mathrm{~N}_{6} \mathrm{O}_{7} \mathrm{~S}_{2} 566.1617$ found $589.1503\left(\mathrm{MNa}^{+}\right)$.

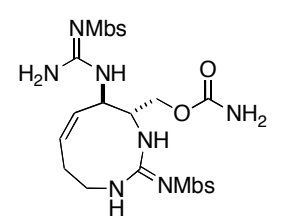

TLC $\mathrm{R}_{f}=0.29\left(10: 1 \mathrm{CH}_{2} \mathrm{Cl}_{2} / \mathrm{MeOH}\right) ;[\alpha]_{\mathrm{Na}}+164^{\circ}\left(\mathrm{c}=2.20\right.$, DMSO); ${ }^{1} \mathrm{H}$ NMR $\left(\mathrm{d}_{6}-\mathrm{DMSO}, 500 \mathrm{MHz}, 80{ }^{\circ} \mathrm{C}\right) \delta$ 7.74-7.64 (m, 4H), 7.03-6.95 (m, 4H), 6.56 (br s, 2H), 6.34 (br s, 2H), 4.66-4.60 (br m, 1H), 4.57-4.53 (m, 2H), 4.04 (dd, $1 \mathrm{H}, J=11.5,3.3 \mathrm{~Hz}), 3.98(\mathrm{dd}, 1 \mathrm{H}, J=11.4,7.8 \mathrm{~Hz}), 3.84(\mathrm{~s}, 3 \mathrm{H}), 3.82(\mathrm{~s}, 3 \mathrm{H}), 3.81-3.79(\mathrm{~m}, 1 \mathrm{H}), 3.42-3.33$ (br m, 2H), 3.10-3.02 (m, 1H), $2.76(\mathrm{br} \mathrm{s}, 1 \mathrm{H}), 2.04(\mathrm{br} \mathrm{s}, 1 \mathrm{H}) \mathrm{ppm} ;{ }^{13} \mathrm{C}$ NMR $\left(\mathrm{d}_{6}\right.$-DMSO, $\left.125 \mathrm{MHz}, 80{ }^{\circ} \mathrm{C}\right) \delta 161.5$, $161.3,159.1,156.6,156.0,136.1,134.9,132.1,128.9,127.7,127.6,113.7,113.3,62.7,56.6,55.6,55.5,49.5,40.0$ (signal obscured by DMSO), $24.7 \mathrm{ppm}$; IR (thin film) v 3436, 3347, 1734, 1597, 1529, 1258, 1133, 1082, $815 \mathrm{~cm}^{-1}$; HRMS (ES ${ }^{+}$) calcd for $\mathrm{C}_{24} \mathrm{H}_{31} \mathrm{~N}_{7} \mathrm{O}_{8} \mathrm{~S}_{2} 609.1676$ found $632.1580\left(\mathrm{MNa}^{+}\right)$. 


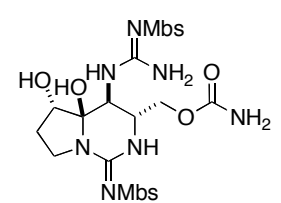

TLC R $\mathrm{R}_{f}=0.21\left(10: 1 \mathrm{CH}_{2} \mathrm{Cl}_{2} / \mathrm{MeOH}\right) ;[\alpha]_{\mathrm{Na}}-120^{\circ}(\mathrm{c}=2.00, \mathrm{MeOH}) ;{ }^{1} \mathrm{H}$ NMR $\left(\mathrm{CD}_{3} \mathrm{CN}, 500 \mathrm{MHz}\right) \delta 7.82-7.77(\mathrm{~m}$, $4 \mathrm{H}), 7.73(\mathrm{~s}, 1 \mathrm{H}), 7.06-7.01(\mathrm{~m}, 4 \mathrm{H}), 6.46($ br s, $2 \mathrm{H}), 6.03($ br d, $1 \mathrm{H}, J=6.1 \mathrm{~Hz}), 5.39($ br s, $2 \mathrm{H}), 4.33(\mathrm{dd}, 1 \mathrm{H}, J=$ 11.8, $2.5 \mathrm{~Hz}), 4.11-4.07(\mathrm{~m}, 1 \mathrm{H}), 3.97(\mathrm{~d}, 1 \mathrm{H}, J=3.8 \mathrm{~Hz}), 3.89(\mathrm{~s}, 3 \mathrm{H}), 3.88(\mathrm{~s}, 3 \mathrm{H}), 3.76(\mathrm{dd}, 1 \mathrm{H}, J=11.8,5.7 \mathrm{~Hz})$, $3.68(\mathrm{ddd}, 1 \mathrm{H}, J=11.1,5.6,2.3 \mathrm{~Hz}), 3.60-3.50(\mathrm{~m}, 2 \mathrm{H}), 2.26-2.18(\mathrm{~m}, 1 \mathrm{H}), 1.86(\mathrm{dd}, 1 \mathrm{H}, J=13.4,7.0 \mathrm{~Hz}) \mathrm{ppm} ;{ }^{13} \mathrm{C}$ NMR $\left(\mathrm{CDCl}_{3}+5 \% \mathrm{CD}_{3} \mathrm{OD}, 125 \mathrm{MHz}\right) \delta 162.4,162.2,156.9,156.5,151.3,134.4,133.8,128.0,127.9,114.0$, $113.9,89.4,74.7,63.8,55.4$ (2), 49.8, 47.2, 46.0, 29.6 ppm; IR(thin film) $v$ 3334, 2924, 1678, 1579, 1536, 1499, 1410, 1260, 1132, 1081, $834 \mathrm{~cm}^{-1}$; HRMS $\left(\mathrm{ES}^{+}\right)$calcd for $\mathrm{C}_{24} \mathrm{H}_{31} \mathrm{~N}_{7} \mathrm{O}_{10} \mathrm{~S}_{2} 641.1574$ found $664.1500\left(\mathrm{MNa}^{+}\right)$.

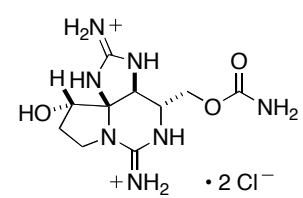

$[\alpha]_{\mathrm{Na}}+126^{\circ}(\mathrm{c}=1.00, \mathrm{MeOH}) ;{ }^{1} \mathrm{H} \mathrm{NMR}\left(\mathrm{D}_{2} \mathrm{O}, 500 \mathrm{MHz}, \mathrm{HOD}\right.$ referenced at $\left.4.80 \mathrm{ppm}\right) \delta 4.77(\mathrm{~d}, 1 \mathrm{H}, J=1.1 \mathrm{~Hz})$, $4.32(\mathrm{~d}, 1 \mathrm{H}, J=4.3 \mathrm{~Hz}), 4.25(\mathrm{dd}, 1 \mathrm{H}, J=11.5,9.2 \mathrm{~Hz}), 4.00(\mathrm{dd}, 1 \mathrm{H}, J=11.6,5.5 \mathrm{~Hz}), 3.82(\mathrm{ddd}, 1 \mathrm{H}, J=9.3,5.4$, $1.1 \mathrm{~Hz}), 3.75(\mathrm{ddd}, 1 \mathrm{H}, J=10.2,10.2,2.0 \mathrm{~Hz}), 3.65(\mathrm{ddd}, 1 \mathrm{H}, J=9.7,9.7,8.3 \mathrm{~Hz}), 2.40(\mathrm{dddd}, 1 \mathrm{H}, J=14.7,9.9$, 9.8, 4.4 Hz), 2.22 (ddd, $1 \mathrm{H}, J=14.8,8.0,1.7 \mathrm{~Hz}) \mathrm{ppm} ;{ }^{13} \mathrm{C} \mathrm{NMR}\left(\mathrm{D}_{2} \mathrm{O}, 125 \mathrm{MHz}\right) \delta 158.6,157.3,155.4,83.0,74.0$, 62.7, 57.4, 52.5, 43.4, 28.4 ppm; HRMS $\left(\mathrm{ES}^{+}\right)$calcd for $\mathrm{C}_{10} \mathrm{H}_{17} \mathrm{~N}_{7} \mathrm{O}_{3} 283.1393$ found $284.1472\left(\mathrm{MH}^{+}\right)$.

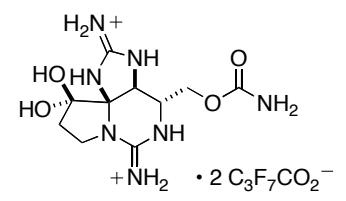

$[\alpha]_{\mathrm{Na}}+84.8^{\circ}\left(\mathrm{c}=0.30\left(\mathrm{CF}_{3} \mathrm{CO}_{2}^{-}\right.\right.$salt $), \mathrm{MeOH}$; literature $\left.\left(\mathrm{Cl}^{-} \text {salt }\right)^{1}+130 \pm 5^{\circ}\right) ;{ }^{1} \mathrm{H} \mathrm{NMR}\left(\mathrm{D}_{2} \mathrm{O}, 500 \mathrm{MHz}, \mathrm{HOD}\right.$ referenced at $4.80 \mathrm{ppm}) \delta 4.72(\mathrm{~d}, 1 \mathrm{H}, J=1.2 \mathrm{~Hz}), 4.27(\mathrm{dd}, 1 \mathrm{H}, J=11.7,9.3 \mathrm{~Hz}), 3.99(\mathrm{dd}, 1 \mathrm{H}, J=11.7,5.4 \mathrm{~Hz})$, $3.80(\mathrm{ddd}, 1 \mathrm{H}, J=9.3,5.3,1.2 \mathrm{~Hz}), 3.76(\mathrm{ddd}, 1 \mathrm{H}, J=10.1,10.1,2.0 \mathrm{~Hz}), 3.56(\mathrm{ddd}, 1 \mathrm{H}, J=9.9,9.9,8.2 \mathrm{~Hz}), 2.41$ $(\mathrm{ddd}, 1 \mathrm{H}, J=13.9,8.3,2.0 \mathrm{~Hz}), 2.32(\mathrm{ddd}, 1 \mathrm{H}, J=14.0,9.9,9.9 \mathrm{~Hz}) \mathrm{ppm} ;{ }^{13} \mathrm{C} \mathrm{NMR}\left(\mathrm{D}_{2} \mathrm{O}, 600 \mathrm{MHz}\right.$, determined by $\mathrm{HMBC}) \delta 159.7,158.2,156.5,99.5,83.1,64.3,57.6,53.7,43.8,33.5$ ppm; HRMS $\left(\mathrm{ES}^{+}\right)$calcd for $\mathrm{C}_{10} \mathrm{H}_{15} \mathrm{~N}_{7} \mathrm{O}_{3}$ 281.1236 found $282.1327\left(\mathrm{MH}^{+}\right)$. 
Comparison of Spectral Data for Synthetic and Natural (+)-Saxitoxin ${ }^{2}$

\begin{tabular}{|c|c|c|c|c|c|}
\hline & \multirow[t]{2}{*}{ Position } & \multicolumn{2}{|c|}{ Synthetic STX } & \multicolumn{2}{|c|}{ Natural STX } \\
\hline & & ${ }^{13} \mathrm{C}$ & ${ }^{1} \mathrm{H}$ & ${ }^{13} \mathrm{C}$ & ${ }^{1} \mathrm{H}$ \\
\hline & 2 & 156.5 & - & 156.8 & - \\
\hline & 4 & 83.1 & - & 83.2 & - \\
\hline & 5 & 57.6 & $4.33(\mathrm{~d}, 1 \mathrm{H}, J=1.2 \mathrm{~Hz})$ & 57.8 & $4.33(\mathrm{~d}, 1 \mathrm{H}, J=1.2 \mathrm{~Hz})$ \\
\hline $\mathrm{HO}_{\mathrm{HN}^{-1}} \mathrm{NH}_{13}$ & 6 & 53.7 & 3.42 (ddd, $1 \mathrm{H}, J=9.3,5.3,1.2 \mathrm{~Hz}$ ) & 53.8 & $3.47(\mathrm{ddd}, 1 \mathrm{H}, J=9,6,1.2 \mathrm{~Hz})$ \\
\hline $\mathrm{HO}=12 y^{5}, \cdots-\mathrm{O}_{14} \mathrm{NH}_{2}$ & 8 & 158.2 & - & 158.5 & - \\
\hline${ }_{10} \mathrm{~N} \prod_{+\mathrm{NH}_{2}^{2}}^{2} \mathrm{NH}$ & 10 & 43.8 & $\begin{array}{l}2.02(\text { ddd, } 1 \mathrm{H}, J=13.9,8.3,2.0 \mathrm{~Hz}) \\
1.93(\mathrm{ddd}, 1 \mathrm{H}, J=14.0,9.9,9.9 \mathrm{~Hz})\end{array}$ & 43.8 & $\begin{array}{l}2.00(\mathrm{~m}, 1 \mathrm{H}) \\
1.99(\mathrm{~m}, 1 \mathrm{H})\end{array}$ \\
\hline & 11 & 33.5 & $\begin{array}{l}3.40(\mathrm{ddd}, 1 \mathrm{H}, J=10.1,10.1,2.0 \mathrm{~Hz}) \\
3.17(\mathrm{ddd}, 1 \mathrm{H}, J=9.9,9.9,8.2 \mathrm{~Hz})\end{array}$ & 33.8 & $\begin{array}{l}3.40(\mathrm{dd}, 1 \mathrm{H}, J=10,3 \mathrm{~Hz}) \\
3.18(\mathrm{ddd}, 1 \mathrm{H}, J=11,10,8 \mathrm{~Hz})\end{array}$ \\
\hline & 12 & 99.5 & - & 99.4 & - \\
\hline & 13 & 64.3 & $\begin{array}{l}3.88(\mathrm{dd}, 1 \mathrm{H}, J=11.7,9.4 \mathrm{~Hz}) \\
3.61(\mathrm{dd}, 1 \mathrm{H}, J=11.7,5.4, \mathrm{~Hz})\end{array}$ & 64.0 & $\begin{array}{l}3.88(\mathrm{dd}, 1 \mathrm{H}, J=12,9 \mathrm{~Hz}) \\
3.65(\mathrm{dd}, 1 \mathrm{H}, J=12,6 \mathrm{~Hz})\end{array}$ \\
\hline & 14 & 159.7 & - & 159.7 & - \\
\hline
\end{tabular}

${ }^{1} \mathrm{H}$ NMR Spectrum of Natural STX $\left(270 \mathrm{MHz}, \mathrm{D}_{2} \mathrm{O}\right)^{3}$

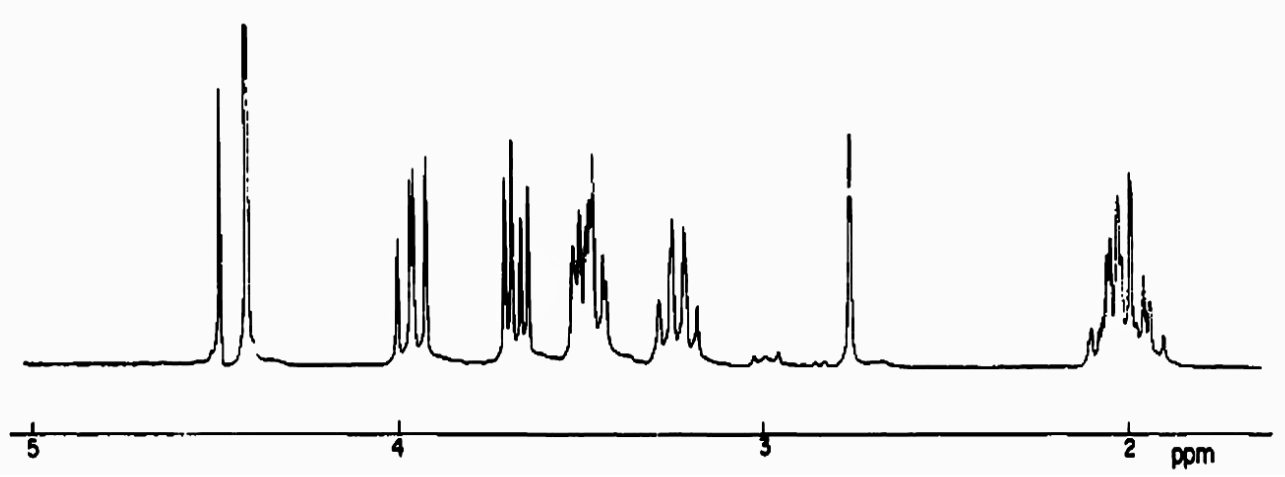

${ }^{1} \mathrm{H}$ NMR Spectrum of Synthetic STX (500 MHz, D 20 , HOD referenced at $\left.4.50 \mathrm{ppm}\right)$

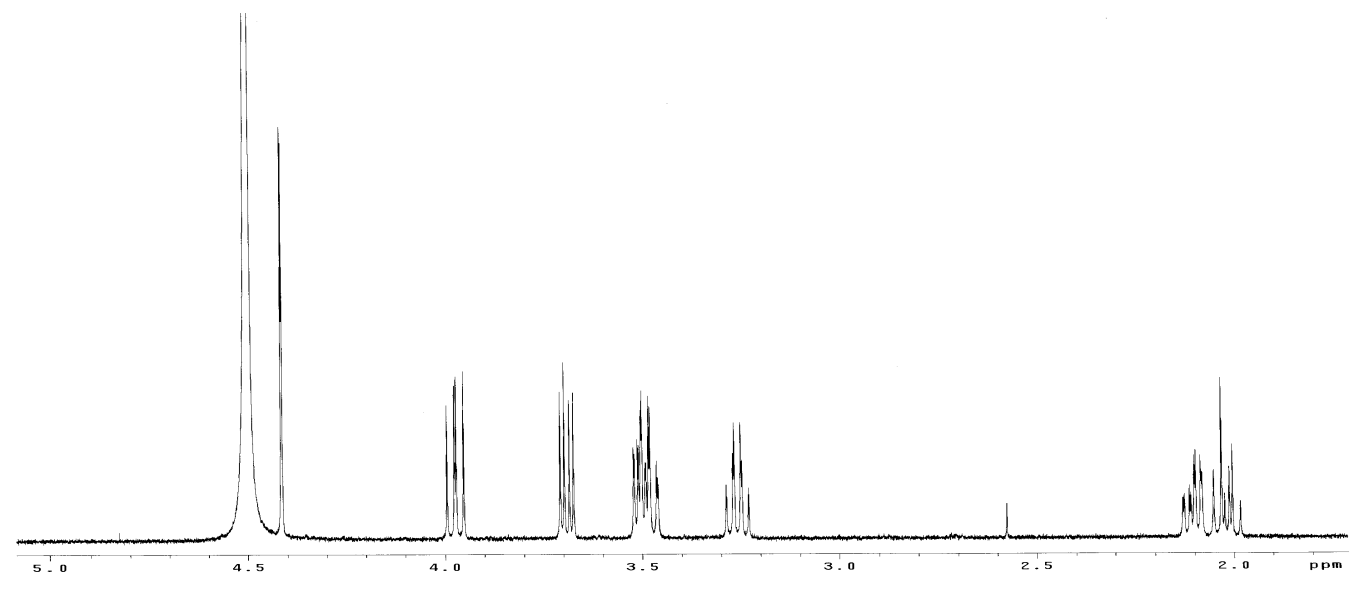

(1) Mold, J. D.; Bowden, J. P.; Stanger, D. W.; Maurer, J. E.; Lynch, J. M.; Wyler, R. S.; Schantz, E. J.; Riegel, B. J. Am. Chem. Soc. 1957, 79, 5235-5238.

(2) Koehn, F. E.; Ghazarossian, V. E.; Schantz, E. J.; Schones, H. K.; Strong, F. M. Bioorg. Chem. 1981, 10, $412-428$.

(3) Niccolai, N.; Schones, H. K.; Gibbons, W. A. J. Am. Chem. Soc. 1980, 102, 1513-1517. 


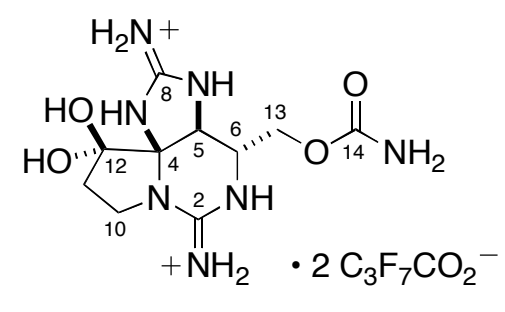

synthetic (+)-saxitoxin

(500 MHz, D $\mathrm{D}_{2} \mathrm{O}$ )

\begin{tabular}{|c|c|c|c|c|c|c|c|c|c|}
\hline 11 & 7 & 7 & 1 & 1 & $T$ & $T$ & T & ? & \\
\hline 8 & 7 & 6 & 5 & 4 & 3 & 2 & 1 & -0 & $\mathrm{ppm}$ \\
\hline
\end{tabular}

